\title{
1933/1945/2012 - Konflikte zwischen „Musikverbrauchern“ und Verwertungsgesellschaften im historischen Kontext
}

\author{
Malte Zill"
}

\section{2012 - die GEMA und das „Clubsterben“"}

Die ökonomische Vertretung von Urheberrechten durch Verwertungsgesellschaften sorgt immer wieder für gesellschaftlichen Unmut. Zuletzt formierte sich 2012 lautstarker Protest gegen die GEMA, nachdem diese ihre Tarifstruktur grundlegend umgestaltet hatte. Nach Gerüchten, dass diese neue Gebührenordnung eine Schließung des traditionsreichen Berliner Clubs Berghain zur Folge haben könnte, vereinten sich DJs, Gastronom:innen und Lobbyist:innen zu einer bundesweiten Protestaktion. Am 25. Juni fanden sich im Berliner Club Frannz mehr als 5.000 Menschen zu einer Demonstration zusammen und warnten mit Transparenten vor einem großangelegten „Clubsterben"1, am 30. Juni stellten Clubs im gesamten Bundesgebiet um 23.55 Uhr für fünf Minuten die Musik ab. ${ }^{2}$ Eine entsprechende Online-Petition wurde in kurzer Zeit mehr als 300.000 Mal unterschrieben. ${ }^{3}$

Die finanzielle Überlastung der Clubs durch Urheberrechtsgebühren und die Sorge vor einem Verlust an kultureller Vielfalt waren meist nur Ausgangspunkte für eine umfassendere Kritik an der GEMA. Überhaupt nütze die Verwertungsgesellschaft nur einer Elite, wahlweise dem „E-Musik-Komponist“ oder dem „Schlagermillionär“, in deren Interesse die GEMA ihre Monopolstellung missbrauche und willkürlich oder zumin-

* Malte Zill, (M.A.) ist Musikwissenschaftler und promoviert an der Universität Hamburg. Sein Forschungsschwerpunkt ist das Urheberrecht Europas zur Zeit des Nationalsozialismus.

1 Twickel, Kalter Krieg im Club, https://www.spiegel.de/kultur/musik/aufruhr-um-ne ue-gema-tarife-und-angst-vor-clubsterben-a-846312.html (zuletzt abgerufen am 4.5.2020).

2 Anonym, Fünf Minuten Saturday Night Stille, https://www.spiegel.de/kultur/musik /neue-gema-tarife-discotheken-fuerchten-clubsterben-und-protestieren-a-841067.ht $\mathrm{ml}$ (zuletzt abgerufen am 4.5.2020).

3 Disselhoff, GEMA: breiter Protest gegen Tarifreform, https://meedia.de/2012/06/27/ gema-breiter-protest-gegen-tarifreform/ (zuletzt abgerufen am 4.5.2020). 
dest intransparent festgelegte Tarife kompromisslos durchsetze. Zudem könne die Verwertungsgesellschaft mit ihren veralteten Methoden im digitalen Zeitalter nicht Schritt halten. Sie sei schlicht nicht kompatibel mit dem um sie herum gewachsenen kulturellen Ökosystem und reagiere darauf mit Repression. ${ }^{4}$

Proteste gegen die Praktiken einer Verwertungsgesellschaft sind keine neue Erscheinung, sondern verweisen auf ein wiederkehrendes gesellschaftliches Akzeptanzproblem des Urheberrechts ${ }^{5}$ und seiner institutionellen Verkörperung. Die GEMA-Tarifreform traf 2012 auf diese generelle Ablehnung, die zudem durch die Wirtschaftskrise und den Veränderungsdruck der Digitalisierung verstärkt wurde. Gerade in gesellschaftlichen und politischen Umbruchszeiten, die meist von wirtschaftlicher Anspannung und rechtlicher Unsicherheit begleitet werden, scheinen das Urheberrecht und seine Verwertung unter besonderen Rechtfertigungszwang zu geraten.

Diese These möchte ich im Folgenden anhand stichprobenartiger Tiefenbohrungen an zwei historischen Zäsuren untersuchen, die auch für die Struktur der Verwertungsgesellschaften prägend waren: die nationalsozialistische Machtübernahme 1933 sowie das Ende des Zweiten Weltkriegs

4 S. Anonym, Gegen die Tarifreform 2013 - GEMA verliert Augenmaß, https://www. openpetition.de/petition/online/gegen-die-tarifreform-2013-gema-verliert-augenma ss\#petition-main (zuletzt abgerufen am 4.5.2020). Vereinzelt finden sich auch von biologistischen Vorstellungen geleitete und deshalb an einen nationalsozialistischen Sprachduktus erinnernde Kommentare (bspw. die GEMA als „unersättliche Zecke am Kulturkörper unserer Zeit"). S. zum Hintergrund Geiger, Musik, 164170. Auch der im Netz viel kommentierte Streit der GEMA mit dem Video-Portal YouTube, der zum Zeitpunkt der GEMA-Proteste von 2012 bereits in vollem Gange war, wurde in den sozialen Medien in ähnlich scharfer Form und mit vergleichbaren Argumenten diskutiert, s. Wragge, Worüber GEMA und YouTube streiten, https://irights.info/artikel/worber-gema-und-youtube-streiten/7182 (zuletzt abgerufen am 4.5.2020), Anonym, Wie geht der Streit zwischen YouTube und Gema weiter?, https://www.cicero.de/innenpolitik/wie-geht-der-streit-zwischen-you tube-und-gema-weiter/49049 (zuletzt abgerufen am 4.5.2020).

5 Die fehlende Akzeptanz des Urheberrechts auf Seite der Konsumierenden ist vielfach besprochen worden. Bemerkenswert ist, dass laut einer Studie der Universität Amsterdam im Auftrag der EU-Kommission von 2006 dies nicht auf unzureichende Kenntnis zurückzuführen ist. Hugenholtz, Amsterdam Law School Research Paper 2012. Auch der Digital-Branchenverband Bitkom diagnostizierte dem Urheberrecht ein solches Akzeptanzproblem. S. BITKOM, Whitepaper: Urheberrecht, https:/www.bitkom.org/sites/default/files/file/import/BITKOM-Whitepaper-Urheb errecht.pdf (zuletzt abgerufen am 4.5.2020). Zur Legitimationskrise urheberrechtlicher Verwertungsgesellschaften aus rechtswissenschaftlicher Perspektive: Denga, Legitimität. 
1945. ${ }^{6}$ Damit möchte ich die Kontinuitäten aufzeigen, die sich ungeachtet dieser historischen Zäsuren in den Argumentationsstrukturen von „Musikverbrauchern" gegenüber den urheberrechtlichen Verwertungsgesellschaften finden lassen. Herauskommen kann dabei selbstverständlich nur ein erster Problemaufriss einer Historisierung gesellschaftlicher Urheberrechtsdebatten. Ausdrücklich möchte ich den Fokus dabei auf die Kontinuitäten des Diskurses zwischen Musiknutzer:innen und der Verwertungsgesellschaft lenken. Weder soll die Frage nach der Berechtigung der Proteste beantwortet, noch die Perspektive der vielfach ebenfalls kritischen Mitglieder eingenommen werden.

Ausgangspunkt der Fallstudie sind historische Beschwerdeschreiben von Gastwirt:innen ${ }^{7}$, regionalen Verbänden und Vereinen gegenüber staatlichen Behörden, die im Bundesarchiv in Berlin sowie im Bayerischen Hauptstaatsarchiv in München überliefert sind. ${ }^{8}$ Diese zeigen deutlich die Rolle von Wirtshäusern und Vereinen bei der politischen Willensbildung ${ }^{9}$ und ein sich daraus ableitendes Mobilisierungspotential, das in unterschiedlichen historischen Konstellationen zu Tage tritt. Gerade in Bezug auf den Aufstieg des Nationalsozialismus und der politischen Sozialisation Hitlers in den Schankkellern Münchens ist diese Funktion als Diskursraum oftmals herausgestellt worden. ${ }^{10}$ Die Grenzen dieser „in Raum und

6 Selbstverständlich würden sich weitere Zäsuren für den Vergleich anbieten. Zu nennen wäre beispielsweise das Ende der deutsch-deutschen Teilung 1990. Hier müsste allerdings die Geschichte der Verwertungsgesellschaft der DDR, der Anstalt zur Wahrung der Aufführungs- und Vervielfältigungsrechte auf dem Gebiet der Musik (AWA), miteinbezogen werden, was an dieser Stelle zu weit führen würde. Weitere mögliche Ansatzpunkte für Recherchen könnten die Ölpreiskrisen der 1970er Jahre, die Terroranschläge der RAF, die Katastrophe von Tschernobyl 1986 oder die Anschläge des 11. Septembers 2001 bieten.

7 Die hier verwendeten Quellen stammen vor 1933 mit einer Ausnahme von männlichen Gastwirten. Die geschlechterspezifische Betonung des Gasthauses als „männlichem Raum“ ist beschrieben worden von Beneder, Männerort Gasthaus. Um die Lesbarkeit und Einheitlichkeit des Textes zu erhalten, sehe ich von der Verwendung des generischen Maskulinums nur für diesen Teil der Quellen ab.

8 Die Dokumente habe ich bei Recherchen gefunden, die ich im Rahmen meines an der Universität Hamburg angesiedelten Dissertationsprojekts Die STAGMA im „Dritten Reich" - Instrumentalisierung des musikalischen Urheberrechts im europäischen Kontext durchführte.

9 In Bezug auf die frühe Neuzeit Dürr, Politische Kultur, 15 f.; Holzem, Kirche, in: Rau, Zwischen Gotteshaus und Taverne, 447; Tlusty, Bacchus; Kümin, The World of the Tavern; aus theoretischer Perspektive Löw, Raumsoziologie, 158 ff.; Günzel, Raum, $97 \mathrm{f}$.

10 Piper, Geschichte, 27. 
Zeit begrenzte[n] Kontaktwelt" ${ }^{\text {"11 }}$ sind von der Rahmensetzung sowohl durch die Gastgeber:innen, als auch durch ordnende Rechtsregelungen abhängig. In den meisten Fällen wird die Rahmensetzung des Rechts durch die Wirt:innen übernommen, etwa hinsichtlich des Besitzes einer Schanklizenz oder der Einhaltung von Hygienevorschriften. Bei einem Verstoß gegen diese rechtliche Rahmensetzung ist mindestens mit dem Wissen und der Akzeptanz der Wirt:innen zu rechnen. Im Falle des Urheberrechts ist jedoch fraglich, ob es in gleicher Weise als eine rechtliche Ordnungsgröße für die Setzung des Diskursrahmens durch die Gastwirt:innen anerkannt oder wie 2012 als Belastung oder Gefahr für den Diskursraum abgelehnt wurde.

\section{1933 - Kritik am Musikschutzverband und die „Machtergreifung“}

Die Wahrnehmung der Urheberrechte übernehmen seit dem Ende des 19. Jahrhunderts die Verwertungsgesellschaften. ${ }^{12}$ Bereits 1898 hatte sich - beeinflusst von der Verabschiedung der Berner Übereinkunft zwei Jahre zuvor $^{13}$ - die Genossenschaft Deutscher Komponisten (später Genossenschaft Deutscher Tonsetzer, kurz GDT) gegründet. 1903 richtete diese mit der Anstalt für musikalisches Aufführungsrecht (AFMA) eine erste Verwertungsgesellschaft ein. Entlang der für die deutschen Verwertungsgesellschaften spezifischen Einteilung von E- und U-Musik zerbrach die Solidargemeinschaft der GDT während des Ersten Weltkriegs. Die Folge war 1915 die Gründung der Genossenschaft zur Verwertung musikalischer Aufführungsrechte (GEMA [alt]), die sich teilweise heftige Konkurrenzkämpfe mit der GDT und der ebenfalls im Deutschen Reich aktiven österreichischen AKM lieferte. Diese unübersichtliche Situation fand 1930 in der Gründung des Verbandes zum Schutze musikalischer Aufführungsrechte für Deutschland, kurz Musikschutzverband (MV), ein vorläufiges Ende, der den drei Gesellschaften einen gemeinsamen Inkassoapparat überstülpte. ${ }^{14}$ Der MV baute ein Netzwerk von Bezirksniederlassungen auf, das Kontrollen in Regionen ermöglichte, die bisher davon ausgenommen waren. Viele der „Musikverbraucher" kamen auf diese Weise zum ersten Mal

11 Wierlacher, Das Gasthaus, in: Wierlacher, Gastlichkeit, 137, 137.

12 Dümling, Musik; Schmidt, Anfänge; Dommann, Autoren, 107-134.

13 Löhr, Globalisierung.

14 S. Dümling, Musik, $164 \mathrm{ff}$. 
mit der Gebührenforderung einer Verwertungsgesellschaft und meist auch dem Urheberrecht in Berührung.

Vertreten wurden die Gastwirt:innen durch das 1928 gegründete Reichskartell der Musikveranstalter. Dieses mischte sich in den Konflikt der Verwertungsgesellschaften ein und nutzte interne Skandale der GEMA [alt], um die Gesellschaft bei den Musikveranstaltern als unmoralisch abzustempeln. ${ }^{15}$ Nach der Vereinigung der Gesellschaften übertrug sich diese generelle Missgunst gegenüber den Urheberrechtsverwertern auf den MV.

Die Machtübernahme der Nationalsozialisten am 30. Januar 1933 wirkte wie ein Katalysator und veranlasste die hoch politisierten Gastwirt:innen zur Agitation gegen den Verband. Der Nationalsozialismus bot die Projektionsfläche für eine erhoffte Erlösung von den Praktiken des MV. Es verwundert daher nicht, dass die Beschwerden in teils drastischer Sprache ideologisch überhöht wurden. Ein 1933 erschienener Artikel, der die Kontrollen des MV behandelte, führte aus, dass es meist Vereinsaufführungen, Standmusiken, Kirchenkonzerte, Volksfeste und Kirchweihen seien, die von den Geldforderungen betroffen waren. Die Aufzählung sollte veranschaulichen, wie weit der MV vom „einfachen Volk“ entfernt war. Die vom Verband „festgesetzten Tantiemen“ würden „rücksichtslos, oft mit Hilfe der Gerichte“ eingetrieben: „Wenn auch das Bestreben der Komponisten auf Erlangung des Lohns für ihre Arbeit anerkannt werden muss", schloss der Artikel, „so ist doch die Art und Weise, mit der die Aufführungen beschnüffelt werden, unsympathisch. " ${ }^{16}$ Immerhin wurde in dem Artikel nicht das Urheberrecht selbst in Frage gestellt, sondern lediglich das Geschäftsgebaren seiner institutionellen Ausprägung.

Diese Mäßigung - die im Wesentlichen der Position des Reichskartells entsprach - teilten die Gastwirt:innen selbst nicht. Sie übernahmen die antisemitischen Positionen der Nationalsozialisten und versuchten, die bestehende Gesellschaft als „nichtarisch” bei den Behörden anzuschwärzen. Ein Auszug aus einem Schreiben des Gastwirts Willi Strübing vom Schützenhaus in Güstrow an das Wirtschaftsministerium kann dies verdeutlichen:

„Das unter der schweren Wirtschaftslage besonders leidende Gewerbe der Gastwirte und Saalbesitzer wird von einer Gesellschaft, die sich

15 Eine Buchhalterin hatte seit 1924 insgesamt 350.000 RM unterschlagen und nach ihrer Entdeckung Selbstmord begangen. Im Abschiedsbrief hatte sie zudem den Leiter des gemeinsamen Büros von AKM und GEMA Hugo Bryk der Mittäterschaft beschuldigt. S. Dümling, Musik $167 \mathrm{f}$.

16 S. Kontrollen des Musikschutzverbandes, Zeitungsnotiz, undatiert, BArch R 55/1151, 27. 
Musikschutzverband betitelt, in einer Weise ausgebeutet, die wohl nicht im Sinne des heutigen Wiederaufbaues liegt [...]. Diese Gesellschaft, die durchaus jüdisch geleitet wird, zwingt uns, an den Musikschutzverband Sonderbeträge für Konzerte u.s.w. zu zahlen [...] Dies sind Lasten [,] die in keiner Weise umzulegen sind und durch die das Gewerbe ruiniert wird. [...] Schließt nun der Saalbesitzer den Vertrag mit der Gesellschaft nicht, weil es ihm gar nicht möglich ist diese Sonderbesteuerung aufzubringen, so wird er durch Kontrolleure besucht. Diese Herren notieren eine jede gespielte [,] noch nicht 30 Jahre alte Komposition und melden dies dem Verband. Derselbe verlangt sodann pro gespieltes Stck [sic] M. 10,- und schreitet sofort zur Klage [...]. Der Endesunterzeichnete ${ }^{17}$ erlaubt sich daher die Anfrage, ob der heutigen nationalen Regierung dieser Überstand bekannt ist und in welcher Weise sie gegen diese, noch aus sozialistischen Zeiten stammende Schöpfung einzuschreiten gedenkt." ${ }^{18}$

Die Beschwerde ging von einer konkreten Gebührenforderung aus, die als zu hoch und oft als existenzbedrohend bezeichnet wurde. Die Wirt:innen brachten daher in der Regel ihre prekäre wirtschaftliche Lage ins Spiel. In vielen Fällen bedienten sie sich zudem des antisemitisch konnotierten Wucher-Vorwurfs oder bezeichneten die Gebührenforderung als Strafe. ${ }^{19}$

Das zweite Beispiel ist ein Ausschnitt aus einem Schreiben des mecklenburgischen Wirtes Wilhelm Toense, der ebenfalls aus finanziellen Gründen seinen Vertrag mit dem MV gekündigt hatte und nun mit Nachforderungen konfrontiert wurde. Dieser brachte einen weiteren bekannten Vorwurf in einer antisemitischen Variante vor - mangelhafte Transparenz und dürftiger Nutzen der Verwertungsgesellschaft für die Urheber:innen:

„Da dieser Konzern vollkommen in jüdischer Hand steckt, wäre ich sehr interessiert zu hören, wieviel die Komponisten für jedes Musikstück erhalten, und wie hoch der Verdienst der Judenbonzen ist. Ich sehe diese ganze Sache als eine reine Erpressung an. Ich bin fest der Meinung, dass Sie auch auf diesem Gebiet Abhilfe schaffen werden." ${ }^{20}$

17 Veraltete Bezeichnung für Unterzeichner.

18 Willi Strübing (Schützenhaus Güstrow) an Min.f. Handel und Wirtschaft, 26.3.1933, BArch R 55/1153, 46.

19 S. GEMA/GDT an Robert Rother, 22.8.1933, BArch R 55/1152, 82; GEMA/GDT an Kathrinchen Niles, 23.8.1933, BArch R 55/1152, 79.

20 Wilhelm Toense an Reichsminister Göring, 30.3.1933, BArch R 55/1152, 124. 
Es wird deutlich, dass die Gastwirt:innen mit der „nationalsozialistischen Revolution" die Hoffnung verbanden, von den als unbillig empfundenen Methoden des MV befreit zu werden. ${ }^{21}$ Mehr als alle anderen Ideologeme des Nationalsozialismus setzte der Antisemitismus den Gesprächsrahmen, in dem die finanziellen Probleme der Gastwirt:innen ausgefochten wurden. Da die Arbeit der Verwertungsgesellschaft die Rolle der Wirtshäuser als Diskursraum gefährdete, verstieß sie gegen den nationalsozialistischen Grundsatz „Gemeinnutz geht vor Eigennutz“ - ein Konflikt, der die Debatten um das Urheberrecht im Nationalsozialismus fortwährend prägte. ${ }^{22}$ Der MV galt daher vielen als Überbleibsel der „Systemzeit“, das es zu beseitigen galt.

Der MV blieb in der Regel unempfänglich für solche Beschwerden und ging konsequent gegen die „Schwarzspieler" vor. Ein Zug vor Gericht war dabei offenbar die Regel, was bis zu Pfändungen eskalieren konnte. ${ }^{23}$ Dieses Verhalten war für die Betroffenen frustrierend und half keineswegs, die Akzeptanz des juristisch gerechtfertigten Anspruchs des MV zu steigern. Im Gegenteil wirkte die Klageerhebung auf die Beklagten bürokratisch und unangemessen ${ }^{24}$, besonders dann, wenn sich die Wirte kooperativ verhielten. ${ }^{25}$

21 Entsprechend schlossen sich auch Parteiorganisationen wie der Kampfbund für deutsche Kultur (KfdK) den Angriffen gegen den MV an und kritisierten die marktwirtschaftliche Ausrichtung der Verwertungsgesellschaft. KfdK an RMVP, 24.8.1933, BArch R 55/1152, $70 \mathrm{f}$. Dagegen betonte das Propagandaministerium die nationalsozialistische Führung des MV, s. RMVP an KfdK, 1.9.1933, BArch R 55/1152, 72 .

22 S. die anonyme Beschwerde eines NSDAP-Mitglieds über die GEMA, 12. 7.1933, BArch R 55/1153, 30. Zur Urheberrechtsdebatte im Nationalsozialismus s. Apel, ZJS 2010, 141; Baldwin, The Copyright Wars, 170-178; Hefti, Das Urheberrecht, in: Dittrich, Woher kommt das Urheberrecht, 165.

23 So im Falle Ferdinand Veiths, Wirt des Gasthauses zum Brockenhof in Eberbach, der den Verband mit einer Gegenklage und anhaltenden Urheberrechtsverletzungen provozierte. S. GEMA/GDT an Ferdinand Veith, 7.8.1933, BArch R 55/1152, 102.

24 Es gibt keine verlässlichen Zahlen über die durch den MV geführten Prozesse. In seiner Autobiographie berichtet der ehemalige Direktor der GDT und spätere Leiter der STAGMA-Vermittlungsabteilung Max Butting von einer Senkung von 22.000 auf 1.200 Prozesse jährlich. S. Butting, Musikgeschichte, 216. Der veröffentlichte Bericht spricht von einer Senkung von 20.000 auf 2.500 Prozesse für das Geschäftsjahr 1933. S. Ritter, Stagma-Nachrichten 1934, 59.

25 Bspw. hatte der Wirt des Schützenhauses in Gössnitz, Rudolf May, zwar mehrmals den Willen zum Vertragsabschluss mit dem MV signalisiert, der diesem jedoch nicht entgegen kam. GEMA/GDT an Rudolf May, 19.8.1933, BArch R 55/1152, 92 . 
Das NS-Regime nutzte die Ablehnung des MV, um die Verwertungsgesellschaften „gleichzuschalten“. Resultat dieser als „Befriedung des Musiklebens" bezeichneten Vereinnahmung war die Gründung der Staatlich genehmigten Gesellschaft zur Verwertung musikalischer Urheberrechte (STAGMA) im September 1933 nach der Verabschiedung des Gesetzes zur Vermittlung von Musikaufführungsrechten vom 4. Juli 1933 („Stagma-Gesetz"). Die Ansprüche der Verwertungsgesellschaft wurden dadurch - anders als es sich die Gastwirt:innen von der „Machtergreifung“ gewünscht hatten - durch das nun zuständige Propagandaministerium gestärkt und die Tantiemen-Verwaltung unter dessen Kontrolle gebracht. ${ }^{26}$

Um die Gemein-/Eigennutzdebatte nicht noch weiter zu verschärfen, versuchte man die Kontinuität, mit der das Verwertungsgesellschaftswesen weitergeführt wurde, zu vertuschen. Dies stellte sich bereits vor der Gründung heraus, als der designierte STAGMA-Direktor Leo Ritter die eingelaufenen Beschwerden damit zurückwies, dass „sich zwar die Situation infolge der Umstellung und Gleichschaltung geändert“ habe, „dass aber die Rechtslage durchaus die gleiche geblieben“" sei. Zusätzlich drehte er die ideologische Argumentation der Beschwerdeführer:innen am Ende des Schreibens um und bemerkte, dass "gerade der neue Staat [...] keine Missachtung gesetzlicher Rechte“, dulde, „weshalb er auch das Urheberrecht in jeder Weise gewahrt wissen will”. ${ }^{27}$ Wohlgemerkt dementierte Ritter den Vorwurf einer Unvereinbarkeit des MV mit dem nationalsozialistischen Regime keineswegs. Vielmehr versuchte auch die STAGMA sich von den Vorgängerorganisationen der "Systemzeit" abzugrenzen.

Hingegen änderte sich aus der Perspektive der Gastwirt:innen kaum etwas am Status Quo. Es verwundert daher nicht, dass einerseits die Beschwerden trotz der STAGMA-Gründung hinaus bis 1935 anhielten ${ }^{28}$ und andererseits im Verlauf des Zweiten Weltkriegs als Folge einer erneut an-

26 Zur „Gleichschaltung“ des MV s. Dümling, Musik, 178-190.

27 GEMA/GDT an Frieda Vehse, 21.8.1933, BArch R 55/1152, 87. Mit derartigen Floskeln arbeitete Ritter eine Vielzahl der hier genannten Beschwerden ab, jeweils inhaltlich und in der Schärfe der Formulierung der Ausgangsbeschwerde angepasst. Ebenfalls wehrte er antisemitische Vorwürfe ab (S. GEMA/GDT an Otto Blome, 16.8.1933, BArch R 55/1152, 98) und verwies auf die „Säuberungen“ des STAGMA-Betriebs: „Was unsere Generalvertretung in Breslau anbetrifft, so können wir Ihnen mitteilen, dass wir bereits die nötigen Maßnahmen zu einer Umorganisation und Gleichschaltung getroffen haben“ (S. GEMA/GDT an Robert Rother, 22.8.1933, BArch R 55/1152, 82).

28 S. verschiedene Anfragen bayerischer Behörden bei vorgesetzten Dienststellen, insbesondere: Bayer. Kultusministerium an RMVP, 26.2.1935, BArch R 55/1154, 208; Bezirksamt Landshut an Regierung von Niederbayern, 28.1.1935, BArch R 
gespannten wirtschaftlichen Situation der Gastwirte zumindest hinter vorgehaltener Hand wieder einsetzten. ${ }^{29}$ Die „Befriedung des Musiklebens“, mit der sich prominente Urheberrechts-Funktionäre wie Richard Strauss und Max Butting schmückten und damit auch ihr Engagement bei der "Gleichschaltung“ der Verwertungsgesellschaften rechtfertigten ${ }^{30}$, ist damit als eine Illusion zu entlarven.

\section{Nach 1945 - Kritik an der Wiederaufnabme der Tätigkeit der STAGMA/ GEMA}

Der Nationalsozialismus lieferte ein Argumentationsgerüst, das je nach den individuellen Interessen der lokalen Akteur:innen gefüllt werden konnte und ganz und gar nicht mit den Vorstellungen in der Hauptstadt übereinstimmen musste. Die genannten Fälle haben deutlich gemacht, dass es stets darum ging, einen ökonomischen Konflikt auf ideologischer Ebene auszutragen. Diese Dynamik wiederholte sich sowohl während des Kriegs als auch nach dessen Ende - nun jedoch unter umgekehrten Vorzeichen.

Auch nach dem Untergang des Nationalsozialismus blieb die Kontinuität zwischen den Verwertungskollektiven gewahrt. Bereits im November 1945 hatte die STAGMA durch den Alliierten Kontrollrat die Genehmigung erhalten, mit dem Einzug von Tantiemen fortzufahren. 1947 nannte sie sich in GEMA um, versah ihre Schreiben jedoch noch mit dem Zusatz „vormals STAGMA“.

Die Kontaktaufnahme der GEMA mit Veranstalter:innen führte wie schon 1933 zu Beschwerden. Generell wurde die Rechtmäßigkeit der Gebührenforderung in Frage gestellt, da die Gastwirt:innen, bisweilen sogar die zuständigen Ministerien selbst, über die Genehmigung durch die Alli-

55/1154, 211. Die STAGMA verfasste daraufhin eine ausführliche Stellungnahme: s. STAGMA an RMVP, 14.3.1935, BArch R 55/1154, 213. Auch die Kontrollen wurden weiter fortgeführt, stachen nur zwischen der stark zunehmenden Überwachung durch Reichsmusikkammer-Vertreter und anderer Akteure des NSÜberwachungsstaates nicht mehr heraus. Zu den Kontrollen der RMK: Fetthauer, „Unerlaubtes“ Musizieren, in: Riethmüller, Reichsmusikkammer, 47; Knickmann Musikforschung 2018, 33.

29 Rößner, Hans, Kritische Äußerungen zur Tätigkeit der Stagma, in: SD-Berichte zu Inlandsfragen, hrsg. v. Chef der Sicherheitspolizei und des SD, Amt III, Berlin, 19.8.1943.

30 S. Butting, Musikgeschichte, 216; Strauss, Späte Aufzeichnungen, 275. 
ierten Kontrollbehörden nicht informiert waren. ${ }^{31}$ Entsprechend waren viele Beschwerdeführer davon ausgegangen, dass die STAGMA ihren Anspruch nach wie vor auf das „Stagma-Gesetz“ von 1933 stützen würde. Die Ablehnung von NS-Kontinuitäten und das Argument finanzieller Überlastung wurden jedoch meist vermischt.

Als Beispiel kann eine Eingabe des Leiters der Musikkapelle Simbach ${ }^{32}$ beim Bayerischen Kultusministerium dienen, die die an die GEMA herangetragenen Vorwürfe besonders anschaulich versammelt:

„Die Stagma wurde in den Zeiten des dritten Reiches ins Leben gerufen. Es handelt sich von jeher um ein Institut, welches einem Finanzamt gleichkommt und alle kulturellen Veranstaltungen schwer hindert, wenn nicht oft ganz unmöglich macht. [...] Man verdammt heute allgemein die Nazi-Diktatur, findet aber daher nicht begreiflich, warum man noch solche vom dritten Reiche errichtete Stellen beibehält. [...] Es ist doch auch jedem klar, dass von den eingehenden Beträgen genau so wie bei manchen anderen Stellen das hauptsächlichste der Verwaltungsapparat aufsaugt. [...] Wann werden einmal die Schlusslichter der Nazi-Diktatur erlischen [sic]? Es wäre wohl höchste Zeit, diese Gehma [sic] genau so wie die Wirtschaftsämter auszublasen.“33

Die Parallelen zu den 1933/34 entstandenen Beschwerden über den MV bzw. die frisch gegründete STAGMA sind kaum zu übersehen. Zunächst

31 Zumindest in Bayern lagen entsprechende Schriftstücke seit dem November 1945 vor. S. die Dokumentensammlung GEMA, November 1945, BayHStA MK 51355; Aldenhoff an Bayerisches Finanzministerium, 23.7.1946, BayHStA MK 51355.

32 Der Widerstand war dabei nicht auf Gastwirte beschränkt. Der Gegenwind für die STAGMA war gerade aus öffentlichen Einrichtungen erheblich. Die Münchener Philharmoniker strebten im Oktober 1946 ein gemeinsames Auftreten mit Radio München und der Generalintendanz der Bayerischen Staatstheater gegenüber der STAGMA an (S. Münchener Philharmoniker an Radio München und Bayerisches Staatstheater, 18.10.1946, BayHStA MK 50807/II). Dort kritisierte man das „bisherige Verhalten der STAGMA“. Da sowieso „fast keine Bühne Zahlungen an die STAGMA leistet“, sollten „auch die Bayer. Staatstheater ihre Zahlungen an die STAGMA einstellen und den Tantiemenbetrag, der auf die STAGMA entfällt, zunächst in Verwahrung nehmen, damit der Betrag haushaltsmäßig später jederzeit zur Verfügung steht“ (S. Vormerkung, An Generaldirektor Dr. Diess, 25.11.1946, BayHStA MK 50807/II). Die Stadt Hannover versuchte noch 1950 mit Hilfe eines Rechtsgutachtens die GEMA-Gebühren für ein Benefizkonzert erstattet zu bekommen. (S. Rechtsgutachten, Stadt Hannover, 19.12.1950, BayHStA MK 50808/I).

33 Hans Wenninger (Leiter der Musikapelle Simbach b. Landau) an Bayer. Kultusministerium, 17.3.1950, BayHStA MK 51356. 
machte der Entstehungskontext der Institutionen in den jeweils vorangegangen und nun als verpönt geltenden Zeiten - sei es die "Systemzeit“ der Weimarer Republik oder die NS-Diktatur - ein Weiterbestehen höchst fragwürdig. Darüber hinaus galt die Verwertungsgesellschaft als Gefahr für das Musikleben, da die „einseitig“ durchgesetzten Tarife als zu hoch angesehen wurden.

Der Landesverein des Bayerischen Gastgewerbes - dessen Schreiben stellvertretend für viele andere Einzelbeschwerden stehen $\mathrm{kann}^{34}$ - warf daher nicht nur die Frage nach der Rechtmäßigkeit des Gebührenanspruchs auf, sondern problematisierte auch den Verbleib jüdischer Tantiemen sowie der Gebühren während des Kriegs besetzter Länder. Weiter forderte man Transparenz bezüglich der seit Kriegsende eingesammelten Gelder. ${ }^{35}$ Auch die Kritik an der behördlichen Struktur ${ }^{36}$, die zudem noch unwirtschaftlich sei, wurde erneut laut. Der Vorwurf der Intransparenz, der 1933 in der Kritik des bürokratischen Verhaltens und mangelnder Aufklärung geäußert wurde, findet sich ebenfalls wieder. Der „Tonkünstler und Vortragsmeister am Flügel“ Leo Vogt-Stolzing aus Rothenburg ob der Tauber warf der STAGMA vor, dass sie ihre Geschäftsbedingungen nicht veröffentliche und es daher für Interpreten unmöglich sei, zu wissen, ob ein Werk bereits gemeinfrei sei. Entsprechend habe die „STAGMA [...] bei vielen, ob Unternehmer oder Künstler nur wenig Freunde" und das Ministerium sei nun gefordert diese „Missstände aus der Welt zu schaffen“.${ }^{37}$ Die

34 Weitere Beschwerden sammelte der Landrat von Ebersberg, der sich im Übrigen die Position der Reklamant:innen zu eigen machte (S. Erschbaumer (Landratsamt Ebersberg) an Regierungspräsident München, 25.3.1946, BayHStA MK 51355). Auch der Oberbürgermeister von Augsburg ersuchte das Ministerium nach Auskunft über die Rechtmäßigkeit der STAGMA-Forderungen gegenüber der Wirtin Anni Michel (s. Oberbürgermeister Augsburg an Bayer. Kultusministerium, 12.6.1946, BayHStA MK 51355; STAGMA an Anni Michel (Hartmannskeller), 3.4.1946, BayHStA MK 51355).

35 S. Landesverband des Bay. Gaststättengewerbes an Bayer. Kultusministerium, 19.7.1946, BayHStA MK 51355.

36 Dieser Vorwurf wurde auch durch das Finanzreferat der Landeshauptstadt München erhoben. Neben der zu verurteilenden Abschöpfung großer Summen durch das Propagandaministerium habe sich die STAGMA nach dem Erlass des Gesetzes vom 4. Juli 1933 wie eine Behörde aufgeführt und von den Finanz- und Steuerbehörden Auskünfte eingefordert. S. Finanzreferat der Landeshauptstadt München an den Oberfinanzpräsident München, 15.4.1947, BayHStA MK 51355.

37 Leo Vogt-Stolzing (Tonkünstler und Vortragsmeister am Flügel, Rothenburg ob der Tauber) an Kulturabteilung der Landesregierung, 5.2.1947, BayHStA MK 51355 . 
STAGMA bzw. GEMA sei nach der Überwindung des NS-Regimes genau wie seinerseits der MV abzuschaffen.

Das Bayerische Kultusministerium sah sich wegen dieser Beschwerden zu einer Untersuchung des Sachverhalts gezwungen. ${ }^{38}$ Trotz der Ablehnung der Gesellschaft und ihrer Praktiken stützte das Ministerium die GEMA $^{39}$ und übernahm sogar deren Argumentation, die STAGMA habe lediglich wirtschaftliche Interessen wahrgenommen, ohne sich in Ideologie zu verstricken. ${ }^{40}$ Das nationalsozialistische Wesen des „Stagma-Gesetzes" wurde dabei ebenso verneint wie jegliche Verantwortung für die Benachteiligung jüdischer Urheber:innen abgewiesen wurde..$^{41}$ Wie 1933 versuchte man, sich schnell von der vergangenen „Systemzeit“ abzugrenzen.

Tatsächlich zeigte die Regierung der BRD durchaus Handlungsbereitschaft zugunsten der Beschwerdeführer:innen. Im März 1952 reichte die Unions-Fraktion im Bundestag eine große Anfrage ein, die sich mit dem Geschäftsgebaren der Verwertungsgesellschaft beschäftigte. Auf der zugehörigen Bundestagsdebatte am 23. April 1952 traten die Vorwürfe noch einmal gebündelt auf. Dem CDU-Abgeordneten Hermann Ehren war vor allem die ungebührliche Tariferhebung von Vereinen ein Dorn im Auge. Ein Kinderfest des Ostvertriebenenvereins Bottrop, dem er selbst vorstand, diente als Beispiel. Zwar bezeichnete er diesen nicht als in seiner Existenz bedroht. Es war ihm aber wichtig, „die großen Organisationen unseres Volkes, die gemeinnützigen Zwecken dienen [...] von einer unhaltbaren Fessel zu befreien". ${ }^{42}$ Aus den anderen Fraktionen kam wenig Widerspruch. Die Ansicht, die GEMA sei bürokratisch, monopolistisch und schade der Kulturvielfalt, wurde von einem breiten Konsens getragen. ${ }^{43}$ Als Er-

38 Bayer. Kultusministerium an Landtagsamt München, 20.1.1950, BayHStA MK 51356.

39 Die einzige Einschränkung des bayerischen Ministeriums bestand in der schwammig formulierten Auflage an die GEMA, dass „bei der Wahrnehmung der an die GEMA übertragenen Inkassorechte ein Verfahren zur Anwendung kommt, das nicht immer wieder zu Klagen Veranlassung gibt“. Bayer. Kultusministerium an Landtagsamt München, 20.1.1950, BayHStA MK 51356.

40 GEMA (vormals STAGMA), Rundschreiben Nr.3, 15.11.1947, BayHStA MK 51355.

41 GEMA (vormals STAGMA) an Bayer. Kultusministerium, 6.11.1947, BayHStA MK 51355.

42 Plenarprotokoll des Deutschen Bundestages, Nr.: 01/205 vom 23.4.1952 http://dip bt.bundestag.de/doc/btp/01/01205.pdf (zuletzt abgerufen am 4.5.2020).

43 Dommann, Autoren, 215. 
gebnis wurde die Staatsaufsicht über Verwertungsgesellschaften ins Auge gefasst, die heute das Deutsche Patent- und Markenamt ausübt. ${ }^{44}$

Die GEMA reagierte auf diese Einschränkung mit einer weiteren rhetorischen Zuspitzung. Sie setzte die Aufführung ohne Lizenz mit dem Tatbestand des Diebstahls gleich ${ }^{45}$ und drehte so an der Eskalationsschraube, die vor dem Hintergrund heutiger Digitalisierungsprozesse in der Kriminalisierung von Filesharing und YouTubern und der stillschweigenden Akzeptanz von Abmahnwellen auf der einen Seite und von alarmierender bis apokalyptischer Rhetorik getragenen Protesten gegen ein "Clubsterben“ auf der anderen Seite weiterrotiert.

\section{Die GEMA - ein anpassungsfähiges Feindbild}

Sowohl bei der „Machtergreifung“ 1933, als auch in der Nachkriegszeit in Zeiten ökonomischer und politischer Spannungen - haben urheberrechtliche Verwertungsgesellschaften mit Gegenwind zu kämpfen gehabt. Die dezentralen Protestwellen 1933 und 1945 verweisen dabei auf die politisierende Funktion von Gasträumen und Vereinen als kulturelle Diskursräume und Echokammern der angenommenen Krise, die wiederum konstituierendes Element der populistisch geführten Debatte um die Verwertungsgesellschaften darstellten.

Die Argumente blieben stets ähnlich und beinhalteten eine Kombination aus Vorwürfen der Intransparenz, Willkür, Kompromisslosigkeit bzw. unlauterem Geschäftsgebaren. Dabei kommt es im Falle der Verwertungsgesellschaften zu einer bemerkenswerten Umkehrung der Zuweisung von Staatlichkeit. Statt gegen den Staat, seine Gesetze und seine Institutionen richtet sich der Protest gegen eine private Organisation, der aber negativ konnotierte staatliche Attribute zugeschrieben werden. Dies findet sich in den genannten Beispielen, in denen der Vorwurf der „Bürokratie“ und der "Willkür“ erhoben wird oder Gebührenforderungen als „Strafe“ oder "Steuer" bezeichnet werden.

Diese Angriffe stellen zudem meist die Vereinbarkeit der Verwertungsgesellschaft mit dem gegenwärtigen Ordnungssystem in Frage, zwischen denen in der Regel ein innerer Widerspruch angenommen wird - also weniger das Urheberrecht selbst als vielmehr dessen Umsetzung in seiner

44 Die Staatsaufsicht wurde durch das Urheberrechtswahrnehmungsgesetz vom 9. September 1965 (BGBl. I S. 1294) eingeführt.

45 Dommann, Autoren, $215 \mathrm{f}$. 
Rechtsstaatlichkeit angezweifelt wird. In den hier vorgestellten Fällen war dies eine überwunden geglaubte politische Ordnung, wie die "Systemzeit“ Weimarer Republik oder das NS-Regime. Die Kritik an der Verwertungsgesellschaft erscheint dann in einem jeweils dem Zeitkontext angepassten ideologischen Gewand und dient der Moralisierung einer populistisch geführten Debatte. In diesem Sinne zeigt die Bundestagsdebatte von 1952 als „Kulturkritik von rechts“46 die Anpassungsfähigkeit des Feindbildes GEMA.

So auch bei den Protesten um die GEMA-Tarifreform von 2012. Auch wenn sich die historische Konstellation offensichtlich von 1933 und 1945 unterscheidet, lassen sich im Konfliktverhalten gegenüber der Verwertungsgesellschaft deutliche Parallelen feststellen. Wieder wurde eine wirtschaftlich als existentiell bezeichnete Bedrohung zum Ausgangspunkt einer polemisch geführten Debatte mit ähnlichen Strukturmerkmalen. Die Vorwürfe der Willkür, der überbordenden Bürokratie und des elitären Duktus wurden erneut genutzt, um der GEMA die Existenzberechtigung abzusprechen. Freilich wurden 2012 demokratische Werte von den Kritiker:innen nicht abgelehnt, im Gegenteil wurde der GEMA ihre elitäre Struktur als ihrerseits undemokratisch angelastet. Die Debatte erfuhr dadurch wiederum eine Moralisierung, die sich in der Anklage wiederfand, die Verwertungsgesellschaft nehme mindestens fahrlässig, wenn nicht mutwillig, die Zerstörung von Kulturvielfalt in Kauf.

Die Frage, ob die Sorgen der Betreiber:innen und Demonstrant:innen gerechtfertigt waren oder nicht, kann an dieser Stelle nicht beantwortet werden. Es sei lediglich darauf hingewiesen, dass der geäußerte Verdacht, die beteiligten Branchenverbände hätten die Proteste mit apokalyptischer Rhetorik angeheizt, um die GEMA in den anstehenden Verhandlungen in Bedrängnis zu bringen ${ }^{47}$, in der Öffentlichkeitsarbeit des Reichskartells der Musikveranstalter und der GDT Ende der 1920er Jahre durchaus einen historischen Vorläufer hatte.

Die Verbände haben jedoch heute - im digitalen Zeitalter - Werkzeuge zur Hand, das Mobilisierungspotential gegenüber den Verwertungsgesellschaften auszuschöpfen. Dies zeigte auch die Auseinandersetzung zwischen der GEMA und YouTube, die parallel zu den Berghain-Protesten ausgefochten wurde. Hier eine finanzielle Überbelastung der beteiligten

46 Ebda.

47 Twickel, Kalter Krieg im Club, https:/www.spiegel.de/kultur/musik/aufruhr-um-n eue-gema-tarife-und-angst-vor-clubsterben-a-846312.html (zuletzt abgerufen am 4.5.2020). 
Akteure durch Tarife anzunehmen ist in Anbetracht ihrer Finanzkraft zumindest fragwürdig. Und auch wenn es zunächst weithergeholt wirken mag, einen multinational agierenden Milliardenkonzern wie Google mit dem Berghain oder gar einem Gasthaus in der deutschen Provinz zu vergleichen, so haben diese dennoch ihre Funktion als Diskursraum gemein, der sich zudem in die Sphäre der sozialen Netzwerke geweitet hat. Die Furcht vor dem Verlust oder der Veränderung dieses Raumes in Verbindung mit den Kommunikationsmöglichkeiten von Social Media gestattet das Wachsen des Protests zu einer Bewegung. Es ist aber auffällig, dass die den Diskursraum bereitstellende Entität oftmals das Urheberrecht als Recht der Künstler:innen auf Vergütung nicht generell ablehnt. Bei der Setzung des Diskursrahmens wird dem Urheberrecht jedoch der Status als ordnende Rechtsregelung abgesprochen.

Diese Beobachtungen sollten nicht dazu führen, die GEMA als Opfer eines generellen Akzeptanzproblems von jeder Verantwortung für die Beständigkeit ihrer Ablehnung freizusprechen. Die historische Einordnung zeigt jedoch, dass die wiederkehrenden Vorwürfe gegenüber der Verwertungsgesellschaft in ihrer polemischen Natur nicht zu einer Übertragung auf praktische Probleme der Verwertungsgesellschaft taugen. Es scheint vielmehr geboten, berechtigte Kritik von politisch motivierten und überspitzten Angriffen zu trennen. ${ }^{48}$ Schon deshalb verlangt der komplexe Gegenstand Verwertungsgesellschaft und deren Wechselwirkungen mit Politik und Gesellschaft eine historisch fundierte wissenschaftliche Beschäftigung.

48 Als Beispiel sei Heinemann, Verteilungspraxis, genannt, dessen Untersuchung über einen rein rechtswissenschaftlichen Blick hinausgeht. 


\section{Literatur}

Anonym, Fünf Minuten Saturday Night Stille, https://www.spiegel.de/kultur/musik /neue-gema-tarife-discotheken-fuerchten-clubsterben-und-protestieren-a-841067. html (zuletzt abgerufen am 4.5.2020).

Anonym, Wie geht der Streit zwischen YouTube und Gema weiter?, https://www.ci cero.de/innenpolitik/wie-geht-der-streit-zwischen-youtube-und-gema-weiter/490 49 (zuletzt abgerufen am 4.5.2020).

Apel, Simon, Das Reichsgericht, das Urheberrecht und das Parteiprogramm der NSDAP, ZJS 1 (2010), S. $141 \mathrm{ff}$.

Baldwin, Peter, The Copyright Wars. Three Centuries of Transatlantic Battle, Princeton 2014.

Beneder, Beatrix, Männerort Gasthaus? Öffentlichkeit als sexualisierter Raum, Frankfurt am Main, 1997.

BITKOM, Whitepaper: Urheberrecht, https://www.bitkom.org/sites/default/files/fil e/import/BITKOM-Whitepaper-Urheberrecht.pdf (zuletzt abgerufen am 4.5.2020).

Butting, Max, Musikgeschichte, die ich miterlebte, Berlin 1955.

Denga, Michael, Legitimität und Krise urheberrechtlicher Verwertungsgesellschaften: kollektive Rechtewahrnehmung zwischen Utilitarismus und Demokratie, Baden-Baden 2015.

Disselhoff, Felix, GEMA: breiter Protest gegen Tarifreform, https://meedia.de/2012/0 6/27/gema-breiter-protest-gegen-tarifreform/ (zuletzt abgerufen am 4.5.2020).

Dommann, Monika, Autoren und Apparate. Die Geschichte des Copyrights im Medienwandel, Frankfurt am Main 2014.

Dümling, Albrecht, Musik hat ihren Wert, Regensburg 2003.

Dürr, Renate, Politische Kultur in der Frühen Neuzeit: Kirchenräume in Hildesheimer Stadt- und Landgemeinden 1550-1750, Heidelberg 2006.

Fetthauer, Sophie, „Unerlaubtes“ Musizieren und Unterrichten: die Ordnungsstrafverfahren der Reichsmusikkammer nach Paragraph 28 der „Ersten Durchführungsverordnung des Reichskulturkammergesetzes“, in: Riethmüller, Albrecht/ Custodis, Michael (Hrsg.), Die Reichsmusikkammer. Kunst im Bann der NaziDiktatur, Köln 2015, S. 47-68.

Geiger, Friedrich, Musik in Zwei Diktaturen. Verfolgung von Komponisten unter Hitler und Stalin, Kassel 2004.

Werlen, Benno, Spatial Turn. Rezeption. Alltägliche Regionalisierung, in: Günzel, Stephan (Hrsg.), Raum. Ein interdisziplinäres Handbuch, Stuttgart 2010, S. 97 f.

Hefti, Ernst, Das Urheberrecht im Nationalsozialismus, in: Dittrich, Robert (Hrsg.), Woher kommt das Urheberrecht und wohin geht es?, Wien 1988, S. 165-180.

Heinemann, Tobias, Die Verteilungspraxis der Verwertungsgesellschaften: Verteilungsmechanismen und wahrnehmungsrechtliche Problemfelder aus einer rechtlichen, ökonomischen, kulturellen und sozialen Sichtweise, Tübingen 2017. 
Holzem, Andreas, Kirche - Kirchhof - Gasthaus. Konflikte um öffentliche Kommunikationsräume in westfälischen Dörfern der Frühen Neuzeit, in: Rau, Susanne (Hrsg.), Zwischen Gotteshaus und Taverne. Öffentliche Räume in Spätmittelalter und Früher Neuzeit, Köln 2003, S. 447-462.

Hugenholtz, P. Bernt et al., The Recasting of Copyright \& Related Rights for the Knowledge Economy, Amsterdam Law School Research Paper 44 (2012).

Knickmann, Tobias, Die ,Strolche‘ der Reichsmusikkammer - Entstehung, Entwicklung und Personal der Berliner Kontrollabteilung 1933-1940“, Die Musikforschung 1 (2018), S. $33 \mathrm{ff}$.

Kümin, Beat (Hrsg.), The World of the Tavern, London 2002.

Löhr, Isabella, Die Globalisierung geistiger Eigentumsrechte: neue Strukturen internationaler Zusammenarbeit, 1886-1952, Göttingen 2010.

Löw, Martina, Raumsoziologie, 1. Aufl., Frankfurt am Main 2001.

Piper, Ernst, Geschichte des Nationalsozialismus, 1. Aufl., Bonn 2018.

Ritter, Leo, Bericht über das erste Arbeitsjahr der Vermittlungsabteilung der STAGMA, Stagma-Nachrichten 4 (1934), S. 59.

Schmidt, Manuela, Die Anfänge der musikalischen Tantiemenbewegung in Deutschland, Berlin 2005.

Strauss, Richard, Späte Aufzeichnungen, Beyer, Marion/May, Jürgen/Werbeck, Walter (Hrsg.), Mainz 2016.

Tlusty, Ann B., Bacchus and Civic Order, Charlottesville 2001.

Twickel, Christoph, Kalter Krieg im Club, https://www.spiegel.de/kultur/musik/aufr uhr-um-neue-gema-tarife-und-angst-vor-clubsterben-a-846312.html (zuletzt abgerufen am 4.5.2020).

Wierlacher, Alois, Das Gasthaus als kulturelle Zwischenwelt, in: Wierlacher, Alois (Hrsg.), Gastlichkeit. Rahmenthema der Kulinaristik, Berlin 2011, S. 137-155.

Wragge, Alexander, Worüber GEMA und Youtube streiten, https://irights.info/artike 1/worber-gema-und-youtube-streiten/7182 (zuletzt abgerufen am 4.5.2020). 
